\title{
DESIGN OF THE SNS CAVITY SUPPORT STRUCTURE*
}

\author{
J. Hogan ${ }^{\dagger}$, G. Ciovati, D. Machie, J. Pitts, J. Preble, W. J. Schneider, K. Smith, L. Turlington \\ Thomas Jefferson National Accelerator Facility, Newport News, Virginia 23606, USA \\ K. Matsumoto, R. Mitchell, Los Alamos National Laboratory, Los Alamos NM USA
}

\begin{abstract}
The Spallation Neutron Source (SNS) facility utilizes a Superconducting linac to provide a $1 \mathrm{GeV} \mathrm{H}^{-}$beam with an average power of $2 \mathrm{MW}$. A critical part of the accelerator structure is the superconducting radio frequency (SRF) niobium cavities, which are housed in a titanium liquid helium pressure vessel. These vessels maintain the operating temperature of 2.1 Kelvin while facilitating the mechanical support, tuning and alignment of the SRF cavities. The design, fabrication and operation of the superconducting support structure will be presented with an emphasis on the design criteria as they pertain to the operation of the cavity. These criteria will be discussed with respect to the titanium liquid helium pressure vessels, higher order mode couplers, fundamental power coupler, field probe and tuning requirements.
\end{abstract}

\section{INTRODUCTION}

In 1999 the SNS management chose to implement superconducting cryomodules within the linac design due in part to the clear advantage over normal conducting at higher power. Since that time Jefferson Lab has developed a design for both the 0.61 beta and the 0.81 beta cryomodules.

The development of the two separate cavity designs along with their support structures includes the interfacing of several key components. The cavity shape [1] along with the power coupler [2], higher order mode couplers and field probe are the reference for the interfacing of the helium vessel and tuner components (Figure 1).

\section{DESIGN}

The niobium-titanium dished heads of the cavity are the attachment points for the helium vessel. The helium vessel design was dictated by a five-atmosphere internal pressure requirement due to the potential upset condition from loss of beamline vacuum and a two-atmosphere external pressure requirement due to the potential upset condition from loss of insulating vacuum. An ASME pressure vessel code analysis was done to define the geometry and thickness of the helium vessel. The parameters pertaining to the ASME code analysis are summarized in Table 1. Titanium was chosen because it matches the thermal contraction of niobium and will have

\footnotetext{
*Work supported by the U.S. Department of Energy under contract DE-AC05-00OR22725

thogan@jlab.org
}

minimal effect on the cavity frequency during cooldown.

Table 1: ASME Code Analysis

\begin{tabular}{|l|l|}
\hline Parameter & Value \\
\hline Internal Pressure & 5 Atmospheres \\
\hline External Pressure & 2 Atmospheres \\
\hline Head Thickness & 0.25 inches \\
\hline Shell Thickness & 0.1875 inches \\
\hline Outside Diameter & 24 inches \\
\hline
\end{tabular}

The requirements for the tuning system are given below in Table 2. The tuner design is based on the SACLAY/DESY design for the TESLA cavities (Figure 2 ). The design was modified to accommodate the larger 805-megahertz SNS cavities. The tuner is mounted on the field probe side of the cavity opposite of the fundamental power coupler.

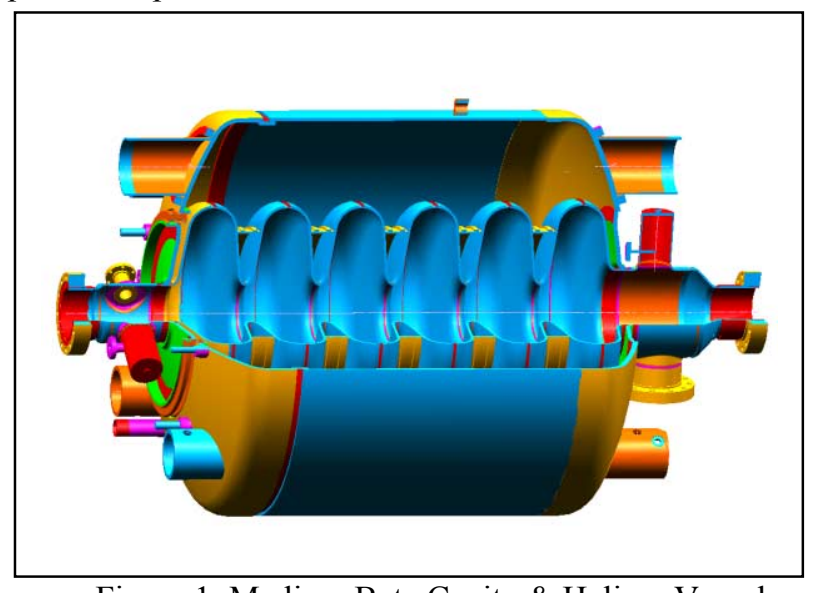

Figure 1. Medium Beta Cavity \& Helium Vessel

The drive system uses a DC stepper motor, with 1.8 degree per step, run through a harmonic drive for reduction of 100:1 and reliability. The tuner and drive system are completely contained within the vacuum space. The tuner bridges a hydro-formed titanium bellows; it is attached to the niobium cavity beamline and the dished head of the titanium helium pressure vessel.

Table 2: SNS Tuner Requirements

\begin{tabular}{|l|l|}
\hline Range & $500 \mathrm{kHz}$ \\
\hline Resolution & $60 \mathrm{~Hz}$ \\
\hline Tuning Coefficient & $200 \mathrm{kHz} / \mathrm{mm}$ \\
\hline Bandwidth & $1,600 \mathrm{~Hz}$ \\
\hline Cavity Spring Constant & $\begin{array}{l}10,000 \\
\text { pounds/inch }\end{array}$ \\
\hline
\end{tabular}




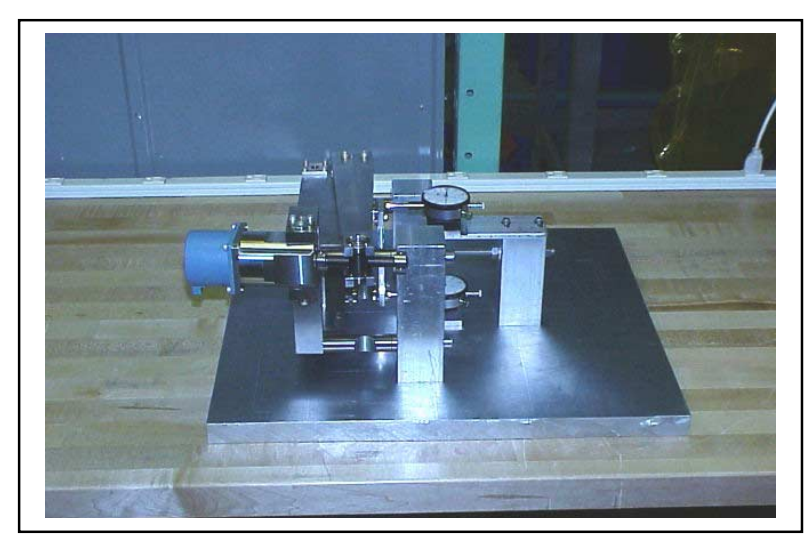

Figure 2. TESLA Tuner

The location and orientation of the power coupler as well as the higher order mode couplers and field probe is critical to the operation of the cavity. Interfacing the helium vessel and tuner with these components was successfully achieved through computer modeling and calculations to discover the optimum location for performance and fabrication.

The support and alignment structure utilize the same double-X pattern of austenitic (Nitronic $\left.{ }^{\circledR} 50\right) 0.5 \mathrm{~cm}$ diameter rods as proven reliable in the CEBAF cryomodule, which has the same diameter helium vessel. Based on modal analysis, the cavity has an additional center support internal to the helium vessel, which anchors the cavity in the transverse directions, yet allows movement along the beamline axis for cooldown and tuning (Figure 3). Each helium vessel is secured in the axial direction via $0.5 \mathrm{~cm}$ Nitronic ${ }^{\circledR} 50$. All of the cavity/helium vessel supports have been designed to compensate for the additional loading due to transportation of the cryomodules from Jefferson Lab to Oak Ridge National Lab (ORNL) [3].

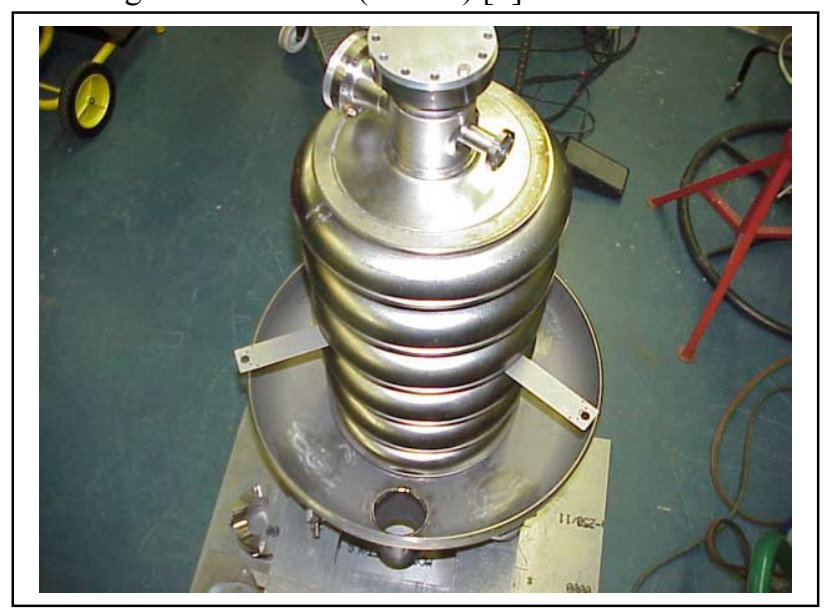

Figure 3. Cavity with Center Supports \& Head

\section{FABRICATION STATUS}

The first prototype cavity and helium vessel have been assembled at Jefferson Lab for testing in June 2001 (Figure 4). Special tooling including an atmospherically isolated glove box is required to attach the cavity to the helium vessel. Due to the Lorentz force detuning effects on the cavity, the helium vessel requires additional stiffening. Testing of the first cavity/helium vessel assembly shall be used to verify computer modeling as well as provide feedback for these modeling technologies.

Three additional 0.61 beta cavities are fabricated and shall be assembled with helium vessels following vertical testing. These next three cavity/helium vessel assemblies shall make up the cavity string for the medium beta prototype cryomodule. This prototype cavity string is scheduled to be completed and rolled out of the clean room for the next assembly stage in the fall of 2001. Once the testing has been completed on the prototype design, all information shall be evaluated and incorporated. Production shall begin in the spring of 2002 with thirty-three medium $(0.61)$ beta cavities followed directly with the forty-eight high $(0.81)$ beta cavities at the rate of one cryomodule per month. Cryomodules shall be cooled down and tested under power prior to shipment to ORNL for compliance with specifications.

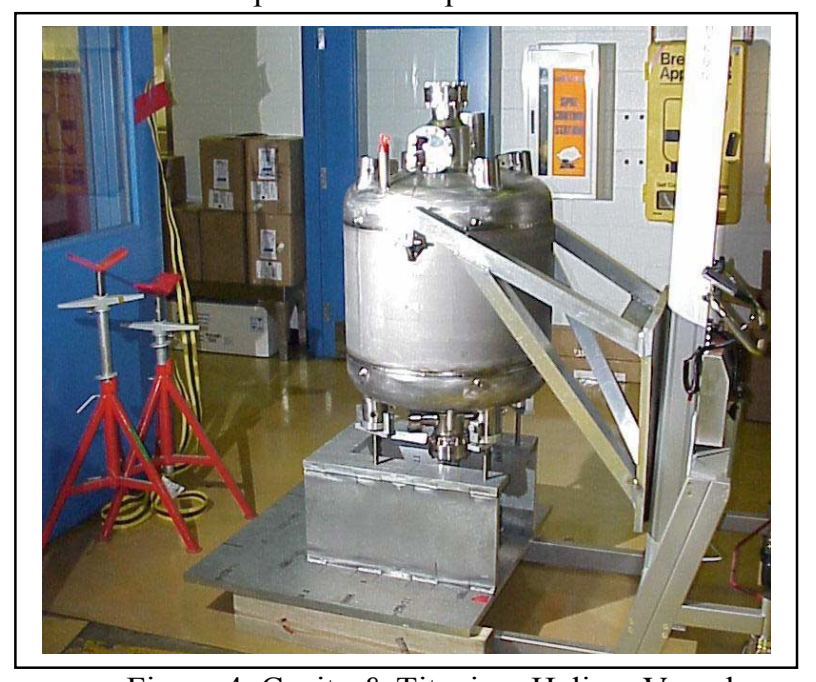

Figure 4. Cavity \& Titanium Helium Vessel

\section{ACKNOWLEDGEMENTS}

- The JLAB mechanical engineering department for their dedication and teamwork.

- The JLAB Superconducting Radio Frequency $\mathrm{R} \& \mathrm{D}$ group for their world-class knowledge and aptitude.

- The JLAB administrative staff for their skill, professionalism and patience.

\section{REFERENCES}

[1] G. Ciovati, et al., "Superconducting Prototype Cavities for the Spallation Neutron Source (SNS) Project,' PAC 2001.

[2] I. E. Campisi, et al., "The Fundamental Power Coupler for the Spallation Neutron Source (SNS) Superconducting Cavities," PAC 2001.

[3] T. Whitlatch, et al., "Shipping and Alignment for the SNS Cryomodule," PAC 2001. 